Cynthia Felicio Batista ${ }^{1}$

Marina Bandeira ${ }^{1}$

Daniela Ramires Oliveira ${ }^{2}$

\section{Fatores associados à sobrecarga subjetiva de homens e mulheres cuidadores de pacientes psiquiátricos}

\author{
Factors associated with the overburden of male and female \\ caregivers of psychiatric patients
}

Laboratório de Pesquisa em Saúde Mental, Universidade Federal de São João del-Rei. Praça Dom Helvécio 74 - Campus Dom Bosco, Bairro das Fábricas. 36300-000 São João del Rei MG Brasil.cynthiafelicio@ yahoo.com.br

2 Departamento de Matemática e Estatística, Universidade Federal de São João del-Rei.

\begin{abstract}
Various studies have investigated the factors associated with the overburden of family caregivers of psychiatric patients. This research sought to identify the factors associated with overburden in groups of male and female caregivers, analyzed separately. Fifty people of each sex participated in the study of family caregivers of psychiatric patients who filled out the Family Overburden Interview Schedule and a standardized sociodemographic data questionnaire. The results showed that different factors were associated with overburden in male and female groups. In the female group, the predictors of a greater degree of overburden were: no children at home, greater number of people living with the family, the patient not performing activities outside the home, the presence of comorbidity and greater incidence of patient problematic behavior. For men, the associated variables were: the family caregiver member not living with the patient, having income, reporting illness resulting from the caregiver role, number of children of the family caregiver, the patient not having a single room at home, patient's age, physical illness and quantity of drugs taken by the patient. These results point to the need for differentiated strategies in the family interventions developed by the mental health services.
\end{abstract}

Key words Mentally-ill persons, Family caregivers, Mental health services
Resumo Estudos têm avaliado os fatores associados à sobrecarga dos familiares cuidadores de pacientes psiquiátricos. A pesquisa visou identificar os fatores associados à sobrecarga subjetiva de grupos de homens e mulheres, analisados separadamente. Participaram da pesquisa 50 homens e 50 mulheres cuidadores de pacientes psiquiátricos que responderam às questões da Escala de Avaliação da Sobrecarga dos Familiares de Pacientes Psiquiátricos e a um questionário sociodemográfico. Os resultados mostraram que diferentes fatores estiveram associados à sobrecarga subjetiva em cada grupo. No grupo de mulheres, os fatores preditores de maior sobrecarga foram: ausência de crianças em casa, maior número de pessoas morando com o familiar, o paciente não desempenhar atividades fora de casa, presença de comorbidade e maior número de comportamentos problemáticos dos pacientes. No grupo de homens, os fatores associados foram: o familiar não morar com o paciente, possuir renda, relatar doença resultante do papel de cuidador, maior número de filhos do familiar, o paciente não possuir quarto individual na casa, ter doença física, idade do paciente e quantidade de medicamentos ingeridos pelo paciente. É necessário adotar abordagens diferenciadas para cada grupo nas ações desenvolvidas pelos serviços de saúde mental.

Palavras-chave Pessoas mentalmente doentes, Cuidadores, Serviços de saúde mental 


\section{Introdução}

A assistência aos pacientes psiquiátricos, baseada em serviços extra-hospitalares, tem resultado em sobrecarga para os seus familiares, tendo em vista que eles se tornaram o principal fornecedor de cuidados cotidianos para estes pacientes, sem terem o preparo para tal e sem orientações efetivas dos serviços de saúde mental, necessárias para o papel de cuidador. Os familiares carecem de esclarecimentos dos profissionais a respeito do transtorno psiquiátrico e de informações acerca de como lidar com os comportamentos problemáticos dos pacientes na vida cotidiana e como agir nos momentos de crise ${ }^{1-3}$.

O conceito de sobrecarga envolve duas dimensões, objetiva e subjetiva. A sobrecarga objetiva se refere às consequências negativas concretas e observáveis resultantes do papel de cuidador, incluindo a frequência de tarefas cotidianas para cuidar dos pacientes e supervisionar seus comportamentos problemáticos, as interrupções na vida social e profissional dos cuidadores e as perdas financeiras. A sobrecarga subjetiva se refere à reação emocional do familiar em relação ao papel de cuidador, incluindo o sentimento de incômodo com as tarefas de cuidado e com as mudanças permanentes em sua vida social e profissional, assim como as preocupações com o paciente $e^{4,5}$.

Maurin e Boyd $\mathrm{d}^{5}$ desenvolveram um modelo explicativo da sobrecarga subjetiva dos familiares cuidadores de pacientes psiquiátricos, adotado no presente trabalho. Nesse modelo, a sobrecarga subjetiva seria resultante da interação de três níveis de variáveis: variáveis antecedentes, sobrecarga objetiva e os fatores moduladores. As variáveis antecedentes são aquelas que definem a situação do transtorno psiquiátrico e envolvem: diagnóstico, gravidade dos sintomas, grau de autonomia do paciente, tipo de residência e as características do programa de tratamento no qual o paciente está engajado. Essas variáveis determinam o grau de sobrecarga objetiva, ou seja, a frequência das tarefas cotidianas necessárias para cuidar do paciente e a frequência de supervisões aos seus comportamentos problemáticos. As variáveis antecedentes, juntamente com a sobrecarga objetiva, determinam o grau de sobrecarga subjetiva sentida pelo familiar. O modelo inclui, ainda, os fatores moduladores que podem afetar a relação entre estas variáveis, podendo aumentar ou diminuir os efeitos das demais variáveis sobre o grau de sobrecarga subjetiva. Eles envolvem: o suporte social que o cuidador pode receber, as suas estratégias de enfrentamento, a qualidade da relação entre familiar e paciente, o nível socioeconômico e outros eventos da vida familiar ${ }^{5}$.

As pesquisas iniciais nesta temática, desenvolvidas no período de 1950 a 1980, investigaram principalmente os níveis de sobrecarga dos familiares e os domínios de suas vidas mais afetados pelo papel de cuidador. Estudos posteriores passaram a investigar também a relação entre a sobrecarga e as variáveis sociodemográficas dos pacientes e familiares, assim como as variáveis clínicas dos pacientes ${ }^{6-8}$.

Os estudos realizados sobre a influência do gênero investigaram apenas as diferenças entre homens e mulheres quanto ao grau de sobrecarga sentida no papel de cuidadores ${ }^{9-19}$ e os domínios mais afetados da vida destes dois grupos de cuidadores $^{8}$. Entretanto, em uma busca realizada em periódicos científicos SciELO, Medline e Lilacs, não foram encontrados estudos que tenham avaliado os fatores associados à sobrecarga de cada um destes grupos de cuidadores, analisados separadamente. O presente estudo teve como objetivo identificar quais variáveis estariam relacionadas à sobrecarga subjetiva de homens e de mulheres cuidadores de pacientes psiquiátricos.

\section{Método}

\section{Delineamento}

Trata-se de uma pesquisa quantitativa, avaliativa, do tipo somativa ou de resultados, cujo objetivo é avaliar os efeitos de serviços, programas ou intervenções, conforme a definição de Contandriopoulos et al. ${ }^{20}$ e de Selltiz et al. ${ }^{21}$ Nesse tipo de avaliação, incluem-se a aferição dos efeitos indesejáveis e os custos não-monetários dos serviços de atendimento aos pacientes psiquiátricos, tal como a sobrecarga sentida pelos familiares, como principais provedores de cuidados cotidianos aos pacientes. A aferição da sobrecarga dos familiares constitui um indicador da qualidade dos serviços de atendimento aos pacientes psiquiátricos, inserindo-se assim no contexto da avaliação dos serviços de saúde mental.

A presente pesquisa consistiu em um estudo do tipo correlacional, no qual são investigadas as relações entre variáveis naturais sem a manipulação de variáveis, que no presente estudo foram as variáveis associadas à sobrecarga dos familiares cuidadores. Constitui, ainda, um estudo de corte transversal, no qual as medidas são tomadas em um único momento no tempo. 
inas Gerais. Trata-se de uma amostra não probabilística, do tipo acidental, na qual os sujeitos são selecionados em função de sua presença nos locais do estudo, durante a coleta de dados, segundo definição de Contandriopoulos et al. ${ }^{20}$. O tamanho da amostra foi definido segundo critérios estabelecidos por Snedecor e Cochran ${ }^{22}$ e Resende et al. ${ }^{23}$

Os critérios de inclusão estabelecidos para a população-alvo dos familiares, de onde foi selecionada a amostra, foram: ter mais de 18 anos e ser indicado, pelos profissionais dos serviços, como o principal cuidador dos pacientes. Foram excluídos os familiares que não estavam em condições de responder ou não entendiam as perguntas feitas durante a entrevista. Para os pacientes, os critérios de inclusão foram: ter mais de 18 anos e apresentar diagnóstico do espectro de esquizofrenia ou transtornos de humor, identificados a partir dos prontuários dos serviços. Foram excluídos os cuidadores de pacientes que estavam internados no período da realização das entrevistas. Foram entrevistados todos os familiares que atendiam aos critérios de inclusão e exclusão e que estavam disponíveis no período da coleta de dados.

\section{Instrumentos de medida}

Escala de Avaliação da Sobrecarga dos Familiares de Pacientes Psiquiátricos (FBIS-BR). Essa escala foi desenvolvida originalmente por Tessler e Gamache ${ }^{24}$, com a denominação de Family Burden Interview Scale (FBIS). Ela foi adaptada para o contexto brasileiro por Bandeira et al..$^{25}$, e validada por Bandeira et al. ${ }^{26}$, tendo recebido a sigla FBIS-BR. A FBIS-BR é composta por cinco subescalas que avaliam as sobrecargas objetiva e subjetiva: A) Assistência na vida cotidiana do paciente; B) Supervisão aos comportamentos problemáticos do paciente; C) Gastos financeiros do familiar com o paciente; D) Impacto nas rotinas diárias do familiar; e E) Preocupações do familiar com o paciente. As questões da escala avaliam a sobrecarga dos familiares nos últimos 30 dias.

A sobrecarga objetiva é avaliada pela frequência de assistência dada ao paciente na vida cotidiana, assim como a frequência de supervi- sões dos seus comportamentos problemáticos e de alterações na rotina do familiar. As opções de respostas são: $1=$ nenhuma vez, $2=$ menos que uma vez por semana, $3=$ uma ou duas vezes por semana, $4=$ de três a seis vezes por semana e 5 $=$ todos os dias. A sobrecarga subjetiva é avaliada pelo grau de incômodo sentido pelo familiar ao realizar tarefas cotidianas de cuidado ao paciente e a supervisão dos seus comportamentos problemáticos, o sentimento de peso financeiro, as preocupações com o paciente e as alterações permanentes ocorridas na vida do familiar. As opções de respostas para avaliar o grau de incômodo são: 1 = nem um pouco, 2 = muito pouco, $3=$ um pouco e $4=$ muito. Para a avaliação do peso financeiro e das preocupações, as opções de resposta são: $1=$ nunca, $2=$ raramente, $3=$ às vezes, $4=$ frequentemente e $5=$ sempre ou quase sempre. Para avaliar o grau de alterações permanentes na vida social e profissional, as opções de respostas são: $1=$ nem um pouco, $2=$ muito pouco, $3=$ um pouco e $4=$ muito.

Questionário sóciodemográfico: Este questionário foi aplicado para avaliar as características sociodemográficas e condições de vida dos familiares e as condições sociodemográficas e características clínicas dos pacientes A facilidade de compreensão desse instrumento foi testada em um estudo-piloto.

\section{Coleta de dados}

A escala FBIS-BR foi aplicada individualmente, por meio de entrevistas nas quais as questões eram lidas e anotadas pelo entrevistador. As entrevistas foram agendadas previamente com os familiares, de acordo com sua disponibilidade. Foram realizadas nas casas dos familiares ou nos serviços de saúde mental, por entrevistadores previamente treinados para assegurar a padronização dos procedimentos de aplicação da escala. Foi entrevistado apenas um cuidador para cada paciente. O presente estudo foi aprovado pela Comissão de Ética em Pesquisa envolvendo Seres Humanos da UFSJ, e pelos serviços de saúde mental participantes. As entrevistas foram realizadas de acordo com os parâmetros éticos e os participantes assinaram o Termo de Consentimento Livre e Esclarecido.

\section{Análise de dados}

As análises de dados foram realizadas no SPSS, versão 13.0. Para investigar os fatores associados ao grau de sobrecarga subjetiva, foi feita a Análi- 
se de Regressão Linear Múltipla, separadamente para cada grupo de cuidadores e para cada uma das seguintes categorias de variáveis: características sociodemográficas e condições de vida dos familiares, assim como as variáveis sociodemográficas e clínicas dos pacientes. $\mathrm{Na}$ análise de regressão, a variável dependente foi o grau de sobrecarga subjetiva e as variáveis independentes foram selecionadas previamente, a partir das seguintes análises estatísticas univariadas: o teste $t$ de Student para amostras independentes, para duas variáveis categóricas, a análise de variância ANOVA, para mais de duas variáveis categóricas e a análise de correlação de Pearson, para as variáveis contínuas.

O critério para inclusão das variáveis independentes na análise de regressão foi apresentar valores de $p<0,25$ nos resultados das análises univariadas referentes à sobrecarga subjetiva. Segundo Hosmer e Lemeshou ${ }^{27}$, variáveis que não foram significativas nas análises univariadas, com valor de $p<0,25$ podem se apresentar como fatores significativos na análise multivariada. As variáveis que permaneceram no modelo de regressão foram aquelas que apresentaram um valor de $p<0,05$.

Nas análises multivariadas, não foram incluídas as constantes na construção dos modelos de regressão, tendo em vista as características da escala de medida usada. A escala FBIS-BR não possui o escore 0 como resposta, pois as opções de respostas estão dispostas em uma escala do tipo Likert, em que o valor mínimo é igual a 1 . Segundo Montgomery e Peck ${ }^{28}$, quando não há a possibilidade de resposta zero, a utilização da constante na análise de regressão pode prejudicar o valor explicativo do modelo.

\section{Resultados}

\section{Descrição da amostra}

A maioria dos cuidadores do sexo masculino era casada $(58,00 \%)$ e tinha média de idade de 50,78 anos. Desses familiares, $36,00 \%$ eram pais dos pacientes e $34,00 \%$ eram irmãos. Nesse grupo, $64,00 \%$ dos familiares recebiam ajuda para cuidar dos pacientes.

No grupo das mulheres cuidadoras, a média de idade foi de 52,66 anos e 52,00\% delas eram casadas. Das mulheres cuidadoras, $42,00 \%$ eram cônjuges do paciente e $28,00 \%$ eram irmãs. No grupo das mulheres, $58,00 \%$ recebiam um tipo de ajuda para cuidar dos pacientes.
Quanto às características dos pacientes que estavam sob cuidados destes familiares, no grupo dos homens cuidadores, a maioria dos pacientes era do sexo feminino (64,00\%). A média de idade era de 40,98 anos. A maioria dos pacientes fazia atividade dentro de casa (74,00\%). Quanto às características clínicas, a maioria dos pacientes foi diagnosticada com transtorno do espectro esquizofrênico $(76,00 \%)$ e $86,00 \%$ não possuíam outros transtornos associados. A maior parte desses pacientes não possuía doenças físicas $(60,00 \%)$, não estava em crise $(78,00 \%)$, aceitava tomar a medicação $(92,00 \%)$ e tomava os medicamentos sozinhos $(52,00 \%)$

No grupo das mulheres cuidadoras, a média de idade dos pacientes era de 43,15 anos. A maior parte era do sexo masculino $(58,00 \%)$ e solteira $(68,00 \%)$. A maioria dos pacientes não desenvolvia atividades fora de casa $(62,00 \%)$. Neste grupo das mulheres cuidadoras, a maior parte dos pacientes foi diagnosticada com transtornos do espectro esquizofrênico $(78,00 \%)$, não possuía outros transtornos $(78,00 \%)$, não possuía doenças físicas $(60,00 \%)$, não estava em crise $(84,00 \%)$, aceitava tomar medicação $(78,00 \%)$ e não tomava medicação sozinha (58,00\%). A descrição mais completa da amostra do presente estudo pode ser encontrada no artigo desenvolvido por Batista et al. ${ }^{10}$.

\section{Análises univariadas da sobrecarga global subjetiva}

Serão apresentados, de forma resumida, os resultados das análises estatísticas univariadas. A descrição completa dessas análises encontra-se em Batista ${ }^{29}$. Para o grupo de cuidadores do sexo feminino, foram selecionadas variáveis em três categorias. Na categoria das condições de vida dos familiares, foram selecionadas as seguintes variáveis: número de pessoas que moram com o familiar, número de filhos do familiar, crianças na mesma casa do paciente. Na categoria sociodemográfica dos pacientes, as variáveis selecionadas foram: estado civil e ter atividade fora de casa. Na categoria das variáveis clínicas dos pacientes, foram selecionadas: número de comportamentos problemáticos, número de medicamentos e presença de comorbidade. As variáveis sociodemográficas dos familiares não atingiram os critérios de seleção definidos previamente, portanto não foi selecionada nenhuma variável desta categoria.

Para o grupo dos cuidadores do sexo masculino, foram selecionadas as seguintes variáveis na categoria sociodemográfica do familiar: familiar 
mora com o paciente, familiar possui renda, tipo de parentesco do familiar com o paciente. $\mathrm{Na}$ categoria das condições de vida dos familiares, as variáveis selecionadas foram: número de pessoas que moram com o familiar, número de filhos do familiar, número de outros familiares que precisam de cuidado, existência de outras preocupações, cuidar de outro familiar doente, doença do familiar cuidador, presença de crianças na mesma casa do paciente e receber ajuda para cuidar do paciente. Na categoria sociodemográfica dos pacientes foram selecionadas duas variáveis: o paciente ter quarto individual e idade do paciente. Na categoria das variáveis clínicas dos pacientes as variáveis foram: número de medicamentos, categoria diagnóstica e presença de doenças físicas do paciente.

Algumas variáveis não foram incluídas nas análises, pois possuíam baixa frequência de respostas. Essas variáveis foram: escolaridade do paciente e familiar, tipo de renda do paciente e familiar, valor da renda do paciente e familiar e tipo de ajuda que o familiar recebe para cuidar do paciente.

\section{Análises multivariadas dos fatores associados à sobrecarga global subjetiva}

Serão apresentados, a seguir, os resultados das análises de Regressão Linear Múltipla feitas separadamente para cada grupo de cuidadores e para cada grupo de variáveis independentes.

\section{Grupo de mulheres cuidadoras}

A Tabela 1 apresenta os resultados obtidos nas análises multivariadas, referentes aos fatores associados à sobrecarga subjetiva das mulheres cuidadoras. Os dados são apresentados, separadamente, para cada categoria de variáveis.

Condições de vida dos familiares: Nesta categoria, os fatores associados à sobrecarga subjetiva obtidos na análise de regressão foram: presença de crianças em casa e número de pessoas que moram com o familiar. Os resultados indicaram que a sobrecarga subjetiva das cuidadoras era maior quando não havia crianças morando na mesma casa. Além disso, quanto menor era o número de pessoas que moravam com o familiar cuidador, maior era o seu grau de sobrecarga subjetiva. Essas variáveis explicaram 92\% da interferência dessa categoria no grau de sobrecarga.

Características sociodemográficas dos pacientes: A única variável desta categoria mantida no modelo final de regressão foi referente às atividades fora de casa. A sobrecarga subjetiva das cuidadoras era maior quando os pacientes não desempenhavam atividades fora de casa. Tal variável explicou $90 \%$ da contribuição das variáveis relacionadas a esta categoria.

Características clínicas dos pacientes: $\mathrm{Na}$ análise desta categoria, algumas variáveis foram excluídas devido à sua colinearidade com outras variáveis incluídas no modelo. São elas: número de internações, duração das internações e pre-

Tabela 1. Análise de Regressão Múltipla das características dos familiares e pacientes associadas a sobrecarga global subjetiva no grupo de mulheres cuidadoras.

\begin{tabular}{|c|c|c|c|c|c|c|}
\hline Categorias & Variáveis & Beta & DP & $t$ & $\mathbf{p}$ & \\
\hline \multirow{2}{*}{$\begin{array}{l}\text { Condições de vida } \\
\text { dos familiares }\end{array}$} & 1. Crianças em casa & 0,73 & 0,12 & 10,83 & 0,00 & \multirow{2}{*}{$\begin{array}{l}R^{2}=0,92 \\
F(189,94)=276,21 \\
(p=0,00) \\
{[D W=1,67]}\end{array}$} \\
\hline & $\begin{array}{l}\text { 2. Número de pessoas que moram } \\
\text { com o familiar }\end{array}$ & $-0,27$ & 0,05 & $-3,93$ & 0.00 & \\
\hline $\begin{array}{l}\text { Características } \\
\text { sociodemográficas } \\
\text { dos pacientes }\end{array}$ & 1. Atividade fora de casa & 0,95 & 0,08 & 21,61 & 0,00 & $\begin{array}{l}\mathrm{R}^{2}=0,90 \\
\mathrm{~F}(373,68)=466,92 \\
(\mathrm{p}=0,00) \\
{[\mathrm{DW}=1,30]}\end{array}$ \\
\hline \multirow{2}{*}{$\begin{array}{l}\text { Características clínicas } \\
\text { dos pacientes }\end{array}$} & 1. Comorbidade & 0,67 & 0,12 & 8,59 & 0,00 & \multirow{2}{*}{$\begin{array}{l}\mathrm{R} 2=0,91 \\
\mathrm{~F}(182,30)=240,00 \\
(\mathrm{p}=0,00) \\
{[\mathrm{DW}=1,27]}\end{array}$} \\
\hline & $\begin{array}{l}\text { 2. Número de comportamentos } \\
\text { problemáticos }\end{array}$ & 0,33 & 0,03 & 4,21 & 0,00 & \\
\hline
\end{tabular}


sença de crise atual. Segundo Field, altos níveis de colinearidade das variáveis podem interferir nos valores de beta, o que interfere na significância estatística desses coeficientes ${ }^{28}$. Dessa forma, as variáveis que foram incluídas na análise de regressão foram: número de comportamentos problemáticos, presença de comorbidade e quantidade de medicamentos.

Os resultados da regressão mostraram que apenas duas variáveis desta categoria se mantiveram no modelo final, como fatores associados à sobrecarga subjetiva: presença de comorbidade e número de comportamentos problemáticos. A sobrecarga subjetiva das mulheres cuidadoras era maior quando os pacientes apresentavam um outro diagnóstico associado ao diagnóstico principal. Além disso, quanto maior era o número de comportamentos problemáticos do paciente, maior era a sobrecarga do familiar. Essas duas variáveis explicaram 91\% da influência desta categoria para o grau de sobrecarga subjetiva das cuidadoras.

\section{Grupo de homens cuidadores}

A Tabela 2 apresenta os resultados das análises multivariadas para o grau de sobrecarga subjetiva dos homens cuidadores, para cada categoria de variáveis.

Características sociodemográficas dos familiares: As variáveis desta categoria que foram man- tidas no modelo final de análise de regressão foram: o familiar possuir renda e o familiar morar com o paciente. Os resultados indicaram que a sobrecarga subjetiva dos homens cuidadores era maior quando eles não moravam com os pacientes e quando possuíam renda. Tais variáveis explicaram 95\% da influência desta categoria sobre o grau de sobrecarga subjetiva.

Condições de vida dos familiares: Nesta categoria, também foram excluídas as variáveis que apresentavam colinearidade. Foram elas: número de outros familiares doentes e existência de outras preocupações. A análise de regressão indicou que as variáveis associadas à sobrecarga subjetiva foram: doença relatada pelo familiar e número de filhos do familiar. A sobrecarga subjetiva dos homens cuidadores era maior quando eles relatavam apresentar alguma doença decorrente do fato de cuidar do paciente. Além disso, quanto maior o número de filhos dos familiares cuidadores, maior era o seu grau de sobrecarga subjetiva. Tais variáveis explicaram 93\% da influência das condições de vida sobre o grau de sobrecarga subjetiva.

Características sociodemográficas dos pacientes: Duas variáveis desta categoria se mantiveram no modelo final: o paciente possuir quarto individual e idade do paciente. A sobrecarga subjetiva dos homens cuidadores era maior quando os pacientes não possuíam quarto individual. Além

Tabela 2. Análise de Regressão Múltipla das características dos familiares e pacientes associada a sobrecarga global subjetiva no grupo de homens cuidadores

\begin{tabular}{|c|c|c|c|c|c|c|}
\hline Categorias & Variáveis & Beta & DP & $t$ & $\mathbf{p}$ & \\
\hline \multirow{4}{*}{$\begin{array}{l}\text { Características } \\
\text { sociodemográficas } \\
\text { dos familiares }\end{array}$} & 1. Familiar mora com o paciente & 0,97 & 0,20 & 9,57 & 0,00 & \multirow{4}{*}{$\begin{array}{l}\mathrm{R}^{2}=0,95 \\
\mathrm{~F}(197,53)=450,91 \\
(\mathrm{p}=0,00) \\
{[\mathrm{DW}=2,15]}\end{array}$} \\
\hline & & & & & & \\
\hline & 2. Renda do familiar & 0,18 & 0,18 & 3,18 & 0,00 & \\
\hline & & & & & & \\
\hline \multirow{3}{*}{$\begin{array}{l}\text { Condições de vida } \\
\text { dos familiares }\end{array}$} & 1. Doença relatada pelo familiar & 0,85 & 0,08 & 16,38 & 0,00 & \multirow{3}{*}{$\begin{array}{l}\mathrm{R}^{2}=0,93 \\
\mathrm{~F}(161,60)=272,11 \\
(\mathrm{p}=0,00) \\
{[\mathrm{DW}=1,74]}\end{array}$} \\
\hline & 2 Número de filhos & 017 & 004 & 3.25 & 0.02 & \\
\hline & & & & & & \\
\hline \multirow{4}{*}{$\begin{array}{l}\text { Características } \\
\text { sociodemográficas } \\
\text { dos pacientes }\end{array}$} & 1. Quarto individual & 0,59 & 0,24 & 4,53 & 0,00 & \multirow{4}{*}{$\begin{array}{l}\mathrm{R}^{2}=0,91 \\
\mathrm{~F}(151,85)=206,33 \\
(\mathrm{p}=0,00) \\
{[\mathrm{DW}=2,33]}\end{array}$} \\
\hline & & & & & & \\
\hline & 2. Idade do paciente & $-0,38$ & 0,01 & $-2,88$ & 0,00 & \\
\hline & & & & & & \\
\hline \multirow{3}{*}{$\begin{array}{l}\text { Características clínicas } \\
\text { dos pacientes }\end{array}$} & 1. Doenças físicas & 0,55 & 0,18 & 4,19 & 0,00 & \multirow{3}{*}{$\begin{array}{l}\mathrm{R}^{2}=0,91 \\
\mathrm{~F}(175,94)=237,35 \\
(\mathrm{p}=0,00) \\
{[\mathrm{DW}=1,72]}\end{array}$} \\
\hline & 2. Nùmero de medicamentos & 0,43 & 0,08 & 5,41 & 0,00 & \\
\hline & & & & & & \\
\hline
\end{tabular}


disso, quanto menor era a idade do paciente, maior era o grau de sobrecarga subjetiva. Essas variáveis explicaram 91\% da influência das variáveis sociodemográficas dos pacientes sobre $\mathrm{o}$ grau de sobrecarga subjetiva.

Características clínicas do paciente: As variáveis que apresentaram associação com a sobrecarga subjetiva, nesta categoria, foram: presença de doenças físicas e número de medicamentos. Os resultados mostraram que a sobrecarga subjetiva dos homens cuidadores era maior quando os pacientes apresentavam doenças físicas e quando tomavam maior quantidade de medicação. Estas duas variáveis explicaram $91 \%$ da influência das variáveis clínicas sobre o grau de sobrecarga subjetiva.

\section{Discussão}

Os resultados das análises multivariadas mostraram que diferentes fatores estavam associados à sobrecarga subjetiva no grupo de homens e mulheres cuidadores. No grupo das mulheres, duas variáveis referentes às características clínicas do paciente foram preditoras do grau de sobrecarga subjetiva: maior número de comportamentos problemáticos do paciente e a presença de comorbidade. O resultado referente aos comportamentos problemáticos confirmou os dados da pesquisa de Kumar e Mohanty ${ }^{15}$, na qual os comportamentos dos pacientes também estavam significativamente relacionados a uma sobrecarga maior nas esposas cuidadoras. Os resultados da pesquisa de Garrido e Menezes ${ }^{30}$ também confirmaram que a sobrecarga era mais elevada para familiares cuidadores de pacientes com demência, que apresentavam transtornos de comportamento. Na pesquisa de Barroso et al. ${ }^{31}$, foi constatado igualmente que um maior número de comportamentos problemáticos apresentados pelos pacientes era um fator preditivo de maior grau de sobrecarga subjetiva relatada pelos familiares. A presença de comorbidade foi um fator associado à sobrecarga subjetiva, possivelmente pelo fato de representar uma maior gravidade do quadro clínico do paciente. Nas pesquisas revisadas por Maurin e Boyd ${ }^{5}$, Loukissa ${ }^{1}$, Rose ${ }^{8}$, Bandeira e Barroso $^{7}$ e Awad e Vorungati ${ }^{6}$, a gravidade do quadro clínico do paciente estava igualmente associada a um maior grau de sobrecarga subjetiva.

Quanto à análise das características sociodemográficas dos pacientes, no grupo de mulheres, os resultados mostraram que o fato de o paciente não possuir atividade fora de casa foi o único fa- tor preditor da sobrecarga. Na pesquisa de Gallichio et al. ${ }^{12}$, as mulheres dispensavam mais tempo para cuidar do paciente do que os homens. $\mathrm{O}$ fato de o paciente não exercer uma atividade fora de casa pode aumentar a quantidade de tempo gasto pelas cuidadoras no cuidado ao paciente na vida cotidiana, aumentando a sobrecarga das mulheres cuidadoras. No estudo de Tessler e Gamache $^{4}$, o fato de os pacientes permanecerem muito tempo nas instituições psiquiátricas, de certa forma, diminuiu a necessidade de os familiares auxiliá-los nas atividades da vida cotidiana. No estudo de Martinez et al. ${ }^{17}$, a sobrecarga na assistência na vida cotidiana foi maior quando os pacientes não possuíam ocupação.

No que se refere às condições de vida das mulheres cuidadoras, duas variáveis foram preditoras do grau de sobrecarga subjetiva: ausência de crianças em casa e número de pessoas que moravam com o familiar cuidador. O resultado referente à ausência de crianças em casa não corrobora os dados da pesquisa de Barroso et al. ${ }^{31}$, na qual, ao contrário, a presença de crianças em casa contribuiu para elevar a sobrecarga dos familiares. Nas pesquisas revisadas por Maurin e Boyd ${ }^{5}$ e Rose ${ }^{8}$, também foi encontrado que os familiares que possuíam crianças em casa apresentaram-se especialmente afetados. Entretanto, tais pesquisas não foram desenvolvidas exclusivamente com mulheres cuidadoras, mas, sim, com uma amostra mista de cuidadores de ambos os sexos, o que pode ter contribuído para este resultado. Não sabemos qual teria sido o resultado desses estudos se tivesse sido analisada, separadamente, a amostra de cuidadores do sexo feminino. O tipo de amostra talvez seja, portanto, uma possível explicação para estas divergências.

Para as mulheres cuidadoras, talvez a presença de crianças em casa seja um fator modulador que reduz o grau de sobrecarga subjetiva, pois as crianças podem proporcionar momentos de descontração, aliviando as tensões provocadas pela responsabilidade de cuidar de um paciente psiquiátrico. Outra possível explicação para tal resultado, pode se referir ao fato de que a gravidade do quadro apresentado pelo paciente pode afastar as pessoas do convívio familiar, especialmente as crianças. Quanto ao número de pessoas que moravam na casa, um menor número de pessoas contribuiu para elevar a sobrecarga subjetiva no grupo das mulheres cuidadoras. Morar com menos pessoas pode significar ter menos ajuda e não poder dividir a tarefa de cuidar do paciente, o que, possivelmente, aumentaria o grau de sobrecarga subjetiva. 
No grupo dos homens cuidadores, as análises multivariadas indicaram duas variáveis preditoras, na categoria de sociodemográficas dos familiares: o familiar não morar com o paciente e a indicação de que o familiar possui renda. Segundo Tessler e Gamache ${ }^{4}$, mesmo quando os familiares não moram com os pacientes, eles podem gastar muitas horas e empreender muito esforço para fornecer assistência e suporte aos pacientes, resultando em sobrecarga. Além disso, o fato de o paciente não estar próximo de seu familiar cuidador resultaria em menor controle e supervisão de suas atividades e comportamentos, o que poderia ocasionar maior preocupação dos familiares. Nesta situação, os cuidadores necessitam também, constantemente, de se deslocarem de suas casas para oferecer apoio e suporte aos pacientes quando eles moram fora.

Contrariando tais resultados, nas pesquisas revisadas por Loukissa ${ }^{1}$, os familiares que moravam com os pacientes foram mais afetados. Essa divergência de resultados talvez se explique por diferenças na composição das amostras, pois nas pesquisas citadas por Loukissa ${ }^{1}$, as amostras eram mistas, compostas por homens de mulheres, enquanto, no presente trabalho, este resultado foi característico da subamostra de homens cuidadores. Quanto ao segundo fator associado encontrado (possuir renda), este dado corrobora os resultados do estudo de Barroso et al..$^{31}$. Uma possível explicação seria que os cuidadores, neste caso, tinham a necessidade de conciliar sua profissão com a função de cuidador, tendo assim uma dupla função na família, o que pode aumentar o grau de sobrecarga subjetiva. Esta foi também uma interpretação dada por Barroso?.

Quanto às condições de vida dos familiares, no grupo dos homens cuidadores, foram fatores preditores do grau de sobrecarga subjetiva: ter doença relatada pelo familiar cuidador decorrente do papel de cuidador e ter maior número de filhos. O resultado referente a doenças do cuidador confirma dados da pesquisa de Garrido e Menezes ${ }^{30}$, na qual se observou também uma associação entre o familiar cuidador apresentar pior saúde física e um maior grau de sobrecarga subjetiva. Resultados semelhantes foram encontrados na pesquisa de Barroso et al..$^{31}$, pois em sua amostra os familiares que necessitavam fazer tratamento para problemas de saúde apresentavam maiores níveis de sobrecarga subjetiva. O resultado referente ao maior número de filhos confirma também os dados da pesquisa de Barroso et al. ${ }^{31}$, na qual o familiar possuir filhos foi um dos fatores que mais contribuiu para elevar o grau de sobrecarga subjetiva.
As variáveis sociodemográficas dos pacientes que foram preditoras de maior grau de sobrecarga subjetiva, no grupo de homens, foram: o paciente não possuir quarto individual e menor idade do paciente. A primeira variável confirmou dados do estudo de Barroso9 ${ }^{9}$ O fato de o paciente não possuir um quarto individual pode acarretar um aumento no grau de sobrecarga, pois o paciente pode incomodar mais os familiares, diminuindo a privacidade das outras pessoas que moram na mesma casa. Além disso, os comportamentos perturbadores do paciente durante a noite afetam mais as outras pessoas em casa quando o paciente não possui um quarto individual 9. Quanto à idade do paciente, o presente resultado confirmou dados das pesquisas de Dyck et al. ${ }^{11}$ e Urizar e Maldonado ${ }^{32}$, nas quais os familiares de pacientes mais jovens relataram maior grau de sobrecarga subjetiva. Esses resultados talvez se expliquem pelo fato de os pacientes mais jovens talvez apresentarem condições clínicas menos estabilizadas. Nas pesquisas revisadas por Bandeira e Barroso ${ }^{7}$, a idade também foi uma variável associada ao grau de sobrecarga.

Duas variáveis clínicas dos pacientes foram preditoras de maior grau de sobrecarga subjetiva, no grupo de homens: presença de doenças físicas e maior número de medicamentos. $\mathrm{O}$ resultado do primeiro fator corrobora dados da pesquisa de Garrido e Menezes ${ }^{30}$, na qual foi observado também que uma pior saúde física dos pacientes estava associada a um maior grau de sobrecarga subjetiva dos cuidadores. $\mathrm{O}$ dado referente ao número de medicamentos tomados pelo paciente corrobora os resultados da pesquisa de Barroso ${ }^{9}$. O fato de o paciente possuir uma doença física e a quantidade de medicamentos prescritos podem se relacionar com a gravidade de seu quadro clínico. Como já mencionado anteriormente, já foi constatado que a gravidade do quadro clínico do paciente está frequentemente associada a um maior grau de sobrecarga ${ }^{1,5-8}$. Além disso, supervisionar o comportamento de tomar medicamentos pode resultar em maior grau de sobrecarga quando são muitos os medicamentos prescritos.

O presente estudo apresenta algumas limitações. Trata-se de uma amostra não-probabilística o que pode interferir na generalização dos resultados para a população-alvo. Além disso, trata-se de um estudo correlacional, o que não possibilita o estabelecimento de relações causais entre as variáveis estudadas. Por fim, outra limitação do presente estudo refere-se ao fato de ter sido realizado apenas com cuidadores de pacientes que 
estavam em tratamento, não contemplando os cuidadores de pacientes que não estavam em tratamento e que podem apresentar maiores índices de sobrecarga.

\section{Conclusão}

Os resultados dessa pesquisa possibilitaram um maior entendimento acerca da sobrecarga sentida pelos familiares cuidadores de pacientes psiquiátricos. O estudo dos grupos de homens e mulheres cuidadores, avaliados separadamente, permitiu investigar os fatores associados à sobrecarga familiar em cada grupo, o que contribuiu para a produção de conhecimento mais detalhado na área, tendo em vista a escassez de estudos que investigaram essa temática.

No presente estudo, os fatores associados a um maior grau de sobrecarga subjetiva foram diferentes entre os grupos de homens e mulheres cuidadores. Esses resultados apontam para as distintas formas de reação ao papel de cuidador nestes dois grupos e às dificuldades diferenciadas que apresentam no enfrentamento dessa situação. O reconhecimento dessas diferenças permite identificar, mais detalhadamente, as necessidades específicas dos cuidadores em função do gênero e possibilita que os serviços tenham uma abordagem diferenciada no atendimento desses familiares. A diferenciação dos fatores associados à sobrecarga, no grupo de homens e mulheres cuidadores, aponta para a necessidade dos serviços de saúde mental desenvolverem intervenções mais especificas e efetivas para estes dois grupos de cuidadores.

A sobrecarga dos familiares cuidadores consiste em um dos custos não-monetários dos serviços e o desenvolvimento de intervenções mais efetivas visando diminuir esta sobrecarga serviria para melhorar a qualidade destes serviços, tendo em vista que a aferição da sobrecarga constitui um indicador da qualidade dos serviços. Estudos futuros longitudinais poderiam aprofundar ainda mais a avaliação das diferenças da sobrecarga subjetiva em grupos de homens e mulheres cuidadores e sua evolução no tempo.

\section{Colaboradores}

M Bandeira e CF Batista trabalharam na pesquisa, metodologia, concepção e redação final do artigo. DRC Oliveira auxiliou as primeiras autoras na análise e interpretação dos dados. 
Referências

1. Loukissa AD. Family burden in chronic mental illness: a review of research studies. J Adv Nurs 1995; 21(2):248-255.

2. Farias CA, Lima POC, Ferreira LA, Cruzeiro ALS, Quevedo LA. Sobrecarga de cuidadores de usuários de um centro de atenção psicossocial infanto-juvenil no sul do Brasil. Cien Saude Colet 2014; 19(12):4819-4827.

3. Presotto RF, Silveira M, Delgado PGG, Vasconcelos EM. Experiências brasileiras sobre participação de usuários e familiares na pesquisa em saúde mental. Cien Saude Colet 2013; 18(19):2837-2845.

4. Tessler RC, Gamache GM. Family experiences with mental illness. Westport: Auburn House; 2000.

5. Maurin JT, Boyd CB. Burden of mental illness on the family: a critical review. Arch Psychiatr Nurs 1990 4(2):99-107.

6. Awad AG, Voruganti LN. The burden of schizophrenia on caregiver: a review. PharmacoEconomics 2008; 26(2):149-162.

7. Bandeira M, Barroso SM. Sobrecarga das famílias de pacientes psiquiátricos. J bra psiquiatr 2005; 54(1):3446.

8. Rose LE. Families of psychiatric patients: a critical review and future research directions. Arch Psychiat Nurs 1996; 10(2):67-76

9. Barroso S. Sobrecarga de familiares de pacientes psiquiátricos: avaliação de fatores associados [dissertação]. Belo Horizonte: Universidade Federal de Minas Gerais; 2006.

10. Batista CMF, Bandeira M, Quaglia MAC, Oliveira DCR, Albuquerque EPT. Sobrecarga de familiares de pacientes psiquiátricos: influência do gênero do cuidador. Cad Saúde Colet 2013; 21(4):359-369.

11. Dyck DG, Short R, Vitaliano PP. Predictors of burden and infectious illness in schizophrenia caregivers. Psychosom Med 1999; 61(4):411-419.

12. Gallichio L, Siddigi N, Langenberg P, Baumgarten M. Gender differences in burden and depression among informal caregivers of demented elders in the community. Int J Geriatr Psychiatry 2002; 17(2):154-163.

13. Greenberg JS, Kim HW, Greenley JR. Factors associated with subjective burden in siblings of adults with severe mental illness. Am J Orthopsychiatry 1997; 67(2):231241.

14. Hsiao CY. Family demands, social support and caregiver burden in Taiwanese family caregivers living with mental illness: the role of family caregiver gender. J Clin Nurs 2010; 19(23-24):3494-3503.

15. Kumar S, Mohanty S. Spousal burden of care in schizophrenia. J Indian Acad Appl Psych 2007; 33(2):189-194.

16. Magliano L, Fadden G, Madianos M, Almeida JM, Held T, Guarneri M, Marasco C, Tosini P, Maj M. Burden on the families of patients with schizophrenia: results of the BIOMED I study. Soc Psychiatry Psychiatr Epidemiol 1998; 33(9):405-412.

17. Martinez A, Nadal S, Beperet M, Mendioroz P. The schizophrenic patient and his/her relatives: a process of deep change. An Sist Sanit Navarra 2000; 23(1):101 110.

18. Ricard N, Bonin JP, Ezer H. Factors associated with burden in primary caregivers of mentally ill patients. Int J Nurs Stud 1999; 36(1):73-83.
19. Scazufca M, Menezes PR, Almeida OP. Caregiver burden in an elderly population with depression in Sao Paulo, Brazil. Soc Psychiatry Psychiatr Epidemiol 2002; 37(9):416-422.

20. Contandriopoulos AP, Champagne F, Potvin L, Denis JO, Boyle P. Saber preparar uma pesquisa. São Paulo: Editora Hucitec Abrasco; 1994

21. Selltiz C, Wrightsman L, Cook S, Kidder L. Métodos de pesquisa nas relações sociais. $2^{\mathrm{a}}$ ed. Sao Paulo: EPU, 1987.

22. Snedecor GW, Cochran WG. Metodos estatisticos. México: Editora Continental; 1971.

23. Resende MDV, Sturion JA, Pereira JCD. Tamanho amostral para detecção de diferenças significativas entre tratamentos. [Internet] Bol. Pesq. Fl. 2004; 49:109120 [Citado 18 out 10]. Disponivel em: http://www. cnpf.embrapa.br/publica/boletim/boletarqv/boletim 49/Pag_109_120.pdf

24. Tessler RC, Gamache GM. The Family Burden Interview Schedule - Short Form (FBIS/SF). Armherst: Machmer Hall; 1994

25. Bandeira M, Calzavara MGP, Varella AAB. Adaptação transcultural para o Brasil de uma escala de avaliação da sobrecarga sentida pelos familiares de pacientes psiquiátricos. J Bras Psiquiatr 2005; 54(3):206-214.

26. Bandeira M, Calzavara MGP, Castro I. Estudo de validade da escala de sobrecarga de familiares cuidadores de pacientes psiquiátricos. J Bras Psiquiatr 2008; 57(2):98-104.

27. Hosmer DW, Lemeshou S. Applied logistic regression. New York: John Wiley and Sons; 2000.

28. Montgomery D, Peck EA. Introduction to linear regression analysis. New York: John Wiley and Sons; 1992.

29. Batista CMF. Sobrecarga de familiares de pacientes psiquiátricos: influência do gênero do cuidador [dissertação]. São João Del-Rei: Universidade Federal de São João del-Rei; 2012

30. Garrido R, Menezes PR. Impacto em cuidadores de idosos com demencia atendidos em um servico psicogeriatrico. Rev Saude Publ 2004; 38(6):835-841.

31. Barroso SM, Bandeira M, Nascimento E. Sobrecarga de familiares de pacientes psiquiátricos atendidos na rede pública. Rev Psiquiatr Clin 2009; 34(6):270-277.

32. Urízar AC, Maldonado JG. Burden of care in families of patients with schizophrenia. Qual Life Res 2006; 15(4):719-724.

Artigo apresentado em 08/04/2014

Aprovado em 08/12/2014

Versão final apresentada em 10/12/2014 